\title{
Nanoneurobiophysics: new challenges for diagnosis and therapy of neurologic disorders
}

\author{
"...nanotech-based methodologies are mature enough to address \\ many of the problems related to diagnosis and therapy of \\ demyelinating disorders."
}

\begin{abstract}
Keywords: atomic force microscopy $\bullet$ early diagnosis $\bullet$ functionalized nanostructured surfaces $\bullet$ nanobiosensors $\bullet$ nanoscale detection $\bullet$ neurologic diseases
\end{abstract}

The term 'nanoneurobiophysics' has been coined to designate a research area aimed at investigating molecular mechanisms relevant for demyelinating and neurodegenerative disorders and developing new methods based on nanotechnology for diagnosis and therapy of these disorders. A fair question to be asked is whether one could not just simply refer to this type of research as another application of nanotechnology to a medical field. We take the view that introducing a new term is justified since it may help drawing attention to the required integration of various research fields. Significant progress to investigate fundamental mechanisms involved in demyelinating and neurodegenerative disorders can be made when researchers worldwide from different areas fully merge their efforts. On one hand, there is the need of expertise about disorders that can cause nervous system demyelination from the health and life sciences, from medical doctors to biologists and biochemists. On the other hand, physicists, biophysicists, physical chemists are required to unravel the molecular mechanisms involved in the development of such diseases. Once the relevant knowledge at these two levels is available, then professionals in different areas of nanotechnology are called upon to come up with strategies and methods for diagnosis and therapy. With this variety of requirements, significant advances will be more likely if activities are carried out within the same framework represented by nanoneurobiophysics.

The main goals of nanoneurobiophysics can be thus broadly classified into two cat- egories: understand the mechanisms behind neurodisorders at the molecular level, which can only be made by combining experimental, theoretical and simulation methods; develop methodology for prognostics, early diagnostics and therapy of demyelinating and neurodegenerative disorders, for which nanotech methods are crucial, as is the case of nanobiosensors.

\section{Nanobiosensors can be used in the detection of neurologic disorders}

Recent developments in varied areas may be exploited for reaching such goals. For instance, detailed studies with cell membrane models have unveiled some processes of aggregation and formation of fibrils of proteins involved in nervous system diseases. Langmuir monolayers made with phospholipids and other biologically-relevant materials are used to mimic a cell membrane, which is made to interact with pharmaceutical drugs, proteins and other molecules involved in physiological action [1]. By way of illustration, one may mention that beta-amyloid proteins are capable of forming plaques with fibrillar amyloids upon interacting with membranes [2]. Understanding aggregation and its triggering process is crucial to design therapeutic strategies since amyloid formation is related to cell death in the surroundings [3]. The molecular-level pathways leading to neurologic diseases have been investigated in studies with chemical force microscopy, which is a variation of atomic force microscopy (AFM) to measure molecular interaction forces $[4,5]$. This technique is able to
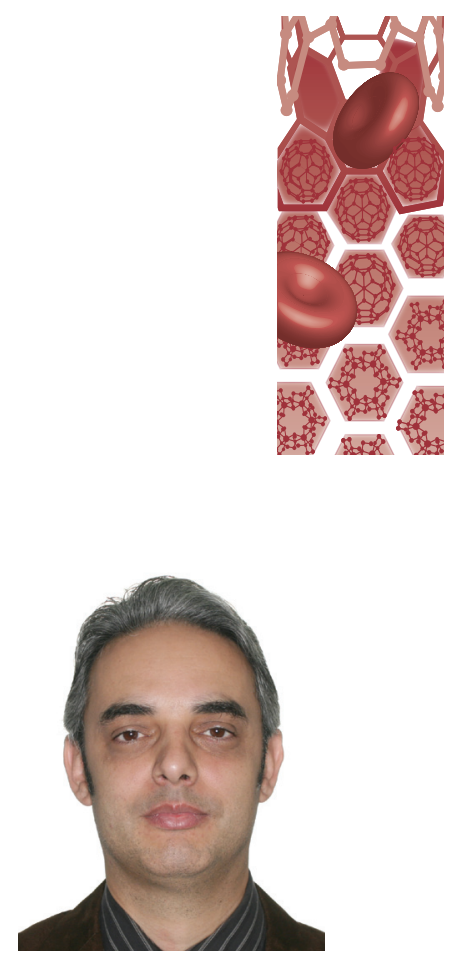

Fabio L Leite

Author for correspondence:

Nanoneurobiophysics Research Group, Department of Physics, Chemistry \& Mathematics, Federal University of São Carlos (UFSCar), Sorocaba, 18052-780, São Paulo, Brazil fabioleite@ufscar.br

Moema Hausen Nanoneurobiophysics Research Group, Department of Physics, Chemistry \& Mathematics, Federal University of São Carlos (UFSCar), Sorocaba, 18052-780, São Paulo, Brazil

Guedmiller S Oliveira Nanoneurobiophysics Research Group, Department of Physics, Chemistry \& Mathematics, Federal University of São Carlos (UFSCar), Sorocaba, 18052-780, São Paulo, Brazil

Doralina G Brum Neurology, Psychology \& Psychiatry Department, Medical College of Botucatu, State University of São Paulo (UNESP), São Paulo, Brazil

Osvaldo N Oliveira Jr São Carlos Institute of Physics, University of São Paulo (USP), São Carlos, SP, Brazil

Future $\%$
Medicine 
detect and diagnose demyelinating diseases before they advance beyond the beginning stages, reducing the chance for false diagnoses [6]. With the combination of AFM and laser scanning confocal microscopy, it is possible to recognize specific molecules responsible for a target disease [7].

\section{"Within the theranostic paradigm, emphasis has been placed on metal nanoparticles that can be labeled with various biomolecules."}

Significant progress has been made toward diagnosis of neurodisorders by using nanostructured films with immobilized biomolecules in biosensors. Molecular recognition between these biomolecules and the biomarkers associated with a disease is exploited to yield high sensitivity and selectivity [8,9]. This strategy has also been highly successful for early detection of cancer by determining small concentrations of biomarkers in the serum [10]. For nervous system disorders, there may be the additional challenge of the low concentration of biomarkers and the specificity required [8]. A possible way to overcome this difficulty is to employ nanobiosensors in which the tip or the cantilever of an AFM is used as the sensing device [11,12]. The tip may be functionalized with a biomolecule for making the sensing very selective, as in an ordinary biosensor. The AFM approach, sometimes referred to as chemical force spectroscopy, is advantageous for various reasons. First, the nanoscopic nature of the device allows for detecting even single molecules [13,14]. Moreover, the actual force between the biomolecule and the analyte, for example, a biomarker, can be measured and related to computational simulations using, for example, steered molecular dynamics $[15,16]$.

Within the theranostic paradigm, emphasis has been placed on metal nanoparticles that can be labeled with various biomolecules. The electrical as well as the fluorescent emission properties of these supramolecular structures can be explored for both detection in screening track identification and for drug release, since these structures would serve as nanocarriers [17]. In diagnosis, antibodies related to neuro-immunopathologies can be immobilized onto nanoparticles. Several challenges must be addressed in order to achieve reliable diagnosis for demyelinating diseases, such as multiple sclerosis (MS) and neuromyelitis optica (NMO), also known as Devic's disease. An effective methodology requires an adequate molecular immobilization protocol, which depends on the cross-linker molecules, surface preparation, chemical reactions, $\mathrm{pH}$ and proper immobilization environment. For instance, immobilization of immunoglobulins demands chemical ligands capable of orienting those molecules so that their binding sites are exposed to the target molecule.

Indirect immunolabeling fluorescence, ELISA and cytometry-based detection assays are the current highly specific methods for diagnosing NMO. However, their sensitivity is still low, ranging from 63 to $76 \%$ [18]. Identification of key molecules such as the aquaporin 4 (AQP4) antibody enables early diagnostics and may allow preventive treatment from the first episode of core clinical characteristics suggestive of NMO, such as optic neuritis, myelitis transverse acute, area postrema syndrome, acute brainstem syndrome and symptomatic narcolepsy $[19,20]$. Considerable improvement in the sensitivity of detection of AQP4 antibody could be made if detection limits reached the nanoscale level. In this context, upon combining experimental procedures with computer-aided molecular design for AFM spectroscopy of functionalized tips, one may achieve pico-level detection. The design is relevant because molecular binding affinity, specificity and other properties such as solubility can be predicted through computational methods, thus avoiding time-consuming experimental procedures [21]. As for MS, there is no diagnostic laboratory test, which often results in delays to reach a definite diagnosis. The identification of suitable biomarkers could radically alter the management of MS at critical phases and allow for prediction of disease development in high-risk populations. This may be achieved with studies involving antibodies to myelin and nonmyelin antigens, cells and soluble molecules of the immune system and analysis of microarray gene expression [22]. So far, no such biomarkers have been discovered.

The examples from the literature mentioned here indicate that nanotech-based methodologies are mature enough to address many of the problems related to diagnosis and therapy of demyelinating disorders. Nevertheless, many challenges are ahead of us. In addition to those related specifically to the study of molecular mechanisms and to developing accurate diagnostics, drug delivery and theranostic systems, there is the main requirement of integrating efforts. Multidisciplinary teams must work in a concerted, seamless fashion to generate the knowledge and technology required to solve the complex problems regarding demyelinating disorders. That is the role of the scientific community of nanoneurobiophysics, which exploits an 'all-inone' concept that connects nanotechnology to various research areas.

\section{Acknowledgements}

The authors thank the students of the Nanoneurobiophysics research group and collaborators for their commitment that makes this research possible. Additional thanks to Dr Luis Peroni from Rhea Biotech for scientific assistance. 
Financial \& competing interests disclosure

This work was supported by FAPESP (07/05089-9, 13/14262-7, 12/50839-4, 13/09746-5, 14/26369-3, 13/14262-7), CAPES/ PNPD 2013/1505 and CNPq. The authors have no other relevant affiliations or financial involvement with any organization

\section{References}

1 Damalio JCP, Nobre TM, Lopes JL, Oliveira ON, Araújo APU. Lipid interaction triggering Septin2 to assembly into $\beta$-sheet structures investigated by Langmuir monolayers and PM-IRRAS. Biochim. Biophys. Acta 1828(6), 1441-1448 (2013).

2 Murphy RM. Kinetics of amyloid formation and membrane interaction with amyloidogenic proteins. Biochim. Biophys. Acta 1768(8), 1923-1934 (2007).

3 Burke KA, Yates EA, Legleiter J. Biophysical insights into how surfaces, including lipid membranes, modulate protein aggregation related to neurodegeneration. Front. Neurol. 4, 17 (2013).

4 Müller DJ, Dufrêne YF. Atomic force microscopy as a multifunctional molecular toolbox in nanobiotechnology. Nat. Nanotechnol. 3(5), 261-269 (2008).

5 Steffens C, Leite FL, Bueno CC, Manzoli A, Herrmann PSDP. Atomic force microscopy as a tool applied to nano/ biosensors. Sensors (Basel) 12(6), 8278-8300 (2012).

6 Lutz R. Nano sensor detects multiple sclerosis in early stages. Webpage report: HCPLive. www.hcplive.com/medical-news

7 Park JW, Park A-Y, Lee S, Yu N-K, Lee S-H, Kaang BK. Detection of TrkB receptors distributed in cultured hippocampal neurons through bioconjugation between highly luminescent (quantum dot-neutravidin) and (biotinylated anti-TrkB antibody) on neurons by combined atomic force microscope and confocal laser scanning microscope. Bioconjug. Chem. 21(4), 597-603 (2010).

8 Epaillard FP. Biosensors applied to the detection of neurodegenerative diseases, a multidisciplinary domain? J. Biosens. Bioelectron. doi:10.4172/2155-6210.1000e103 (2012) (Epub ahead of print).

9 The Handbook of Nanomedicine. Humana Press, NJ, USA. www.springerlink.com

10 Gdowski A, Ranjan AP, Mukerjee A, Vishwanatha JK. Nanobiosensors: role in cancer detection and diagnosis. $A d v$. Exp. Med. Biol. 807, 33-58 (2014).

11 Leite FL, Herrmann PSP. Application of atomic force spectroscopy (AFS) to studies of adhesion phenomena: a review. J. Adhes. Sci. Technol. 19(3-5), 365-405 (2005). or entity with a financial interest in or financial conflict with the subject matter or materials discussed in the manuscript apart from those disclosed.

No writing assistance was utilized in the production of this manuscript.

12 Soto Garcia P, Moreau ALD, Magalhaes Ierich JC et al. A nanobiosensor based on 4-hydroxyphenylpyruvate dioxygenase enzyme for mesotrione detection. IEEE Sens. J. 15(4), 2106-2113 (2015).

13 Zlatanovaa J, Lindsayb SM, Leubac, SH. Single molecule force spectroscopy in biology using the atomic force microscope. Prog. Biophys. Mol. Biol. 74, 37-61 (2000).

14 Leite FL, Bueno CC, Da Róz AL, Ziemath EC, Oliveira ON. Theoretical models for surface forces and adhesion and their measurement using atomic force microscopy. Int. J. Mol. Sci. 13(10), 12773-12856 (2012).

15 Amarante AM, Oliveira GS, Bueno CC et al. Modeling the coverage of an AFM tip by enzymes and its application in nanobiosensors. J. Mol. Graph. Model. 53, 100-104 (2014).

16 Morfill J, Neumann J, Blank K et al. Force-based analysis of multidimensional energy landscapes: application of dynamic force spectroscopy and steered molecular dynamics simulations to an antibody fragment-peptide complex. J. Mol. Biol. 381(5), 1253-1266 (2008).

17 Moghimi SM, Hunter AC, Murray JC. Nanomedicine: current status and future prospects. FASEB J. 19(3), 311-330 (2005).

18 Waters PJ, Pittock SJ, Bennett JL, Jarius S, Weinshenker BG, Wingerchuk DM. Evaluation of aquaporin-4 antibody assays. Clin. Exp. Neuroimmunol. 5(3), 290-303 (2014).

19 Krupp LB, Banwell B, Tenembaum S. Consensus definitions proposed for pediatric multiple sclerosis and related disorders. Neurology. 68(16 Suppl. 2), S7-S12 (2007).

20 Wingerchuk DM, Banwell B, Bennett JL et al. International consensus diagnostic criteria for neuromyelitis optica spectrum disorders. Neurology doi:10.1212/ WNL.0000000000001729 (2015) (Epub ahead of print).

21 Kuroda D, Shirai H, Jacobson MP, Nakamura H. Computeraided antibody design. Protein Eng. Des. Sel. 25(10), 507-521 (2012).

22 Harris VK, Sadiq SA. Disease biomarkers in multiple sclerosis: potential for use in therapeutic decision making. Mol. Diagn. Ther. 13(4), 225-244 (2009). 